\title{
UCRL-TR-228095
}

LA W REN CE LIVERMORE N A TIO N A L LABORATORY
LQCD Phase 1 Runs with P4RHMC

Ron Soltz, Rajan Gupta

February 15, 2007 
This document was prepared as an account of work sponsored by an agency of the United States Government. Neither the United States Government nor the University of California nor any of their employees, makes any warranty, express or implied, or assumes any legal liability or responsibility for the accuracy, completeness, or usefulness of any information, apparatus, product, or process disclosed, or represents that its use would not infringe privately owned rights. Reference herein to any specific commercial product, process, or service by trade name, trademark, manufacturer, or otherwise, does not necessarily constitute or imply its endorsement, recommendation, or favoring by the United States Government or the University of California. The views and opinions of authors expressed herein do not necessarily state or reflect those of the United States Government or the University of California, and shall not be used for advertising or product endorsement purposes.

This work was performed under the auspices of the U.S. Department of Energy by University of California, Lawrence Livermore National Laboratory under Contract W-7405-Eng-48. 


\section{Begin LQCD Phase 1 with p4rhmc}

\section{Background}

These results represent the first set of runs of $10 \beta$ values ranging from 2000 7000 trajectories with the p4rhmc code. This initial run sequence spanned roughly 2-weeks in late January and Early February, 2007.

\section{Scripts}

To manage the submission of dependent jobs

- subSet.pl - submits a set of dependent jobs for a single run

- rmSet.pl - removes a set of dependent jobs in reverse order of submission

- statSet.pl - runs pstat command and prints parsed output along with directory contents

The results of running the statSet.pl command are printed below for three different times during the start up the next sequence of runs using the milc code.

Output of statSet.pl on Wednesday, Feb 07, 2007, 11 AM:

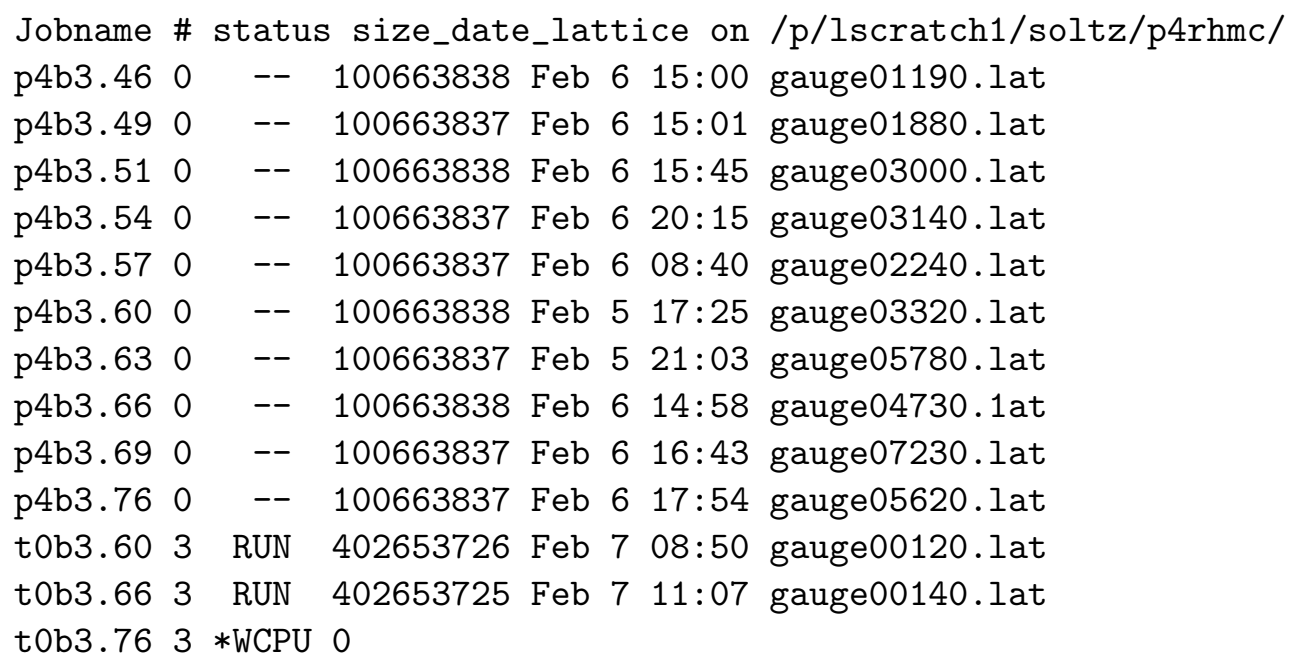




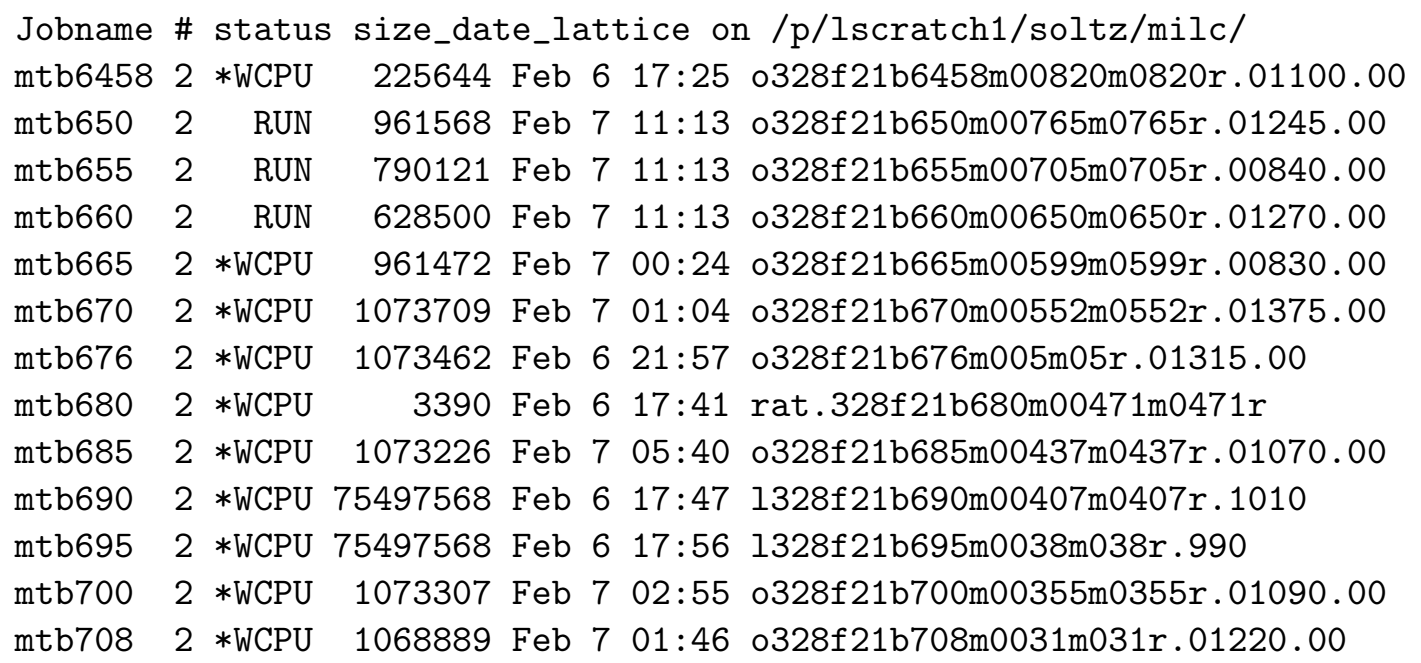

Output of statSet.pl on Wednesday, Feb 07, 2007, 1 PM:

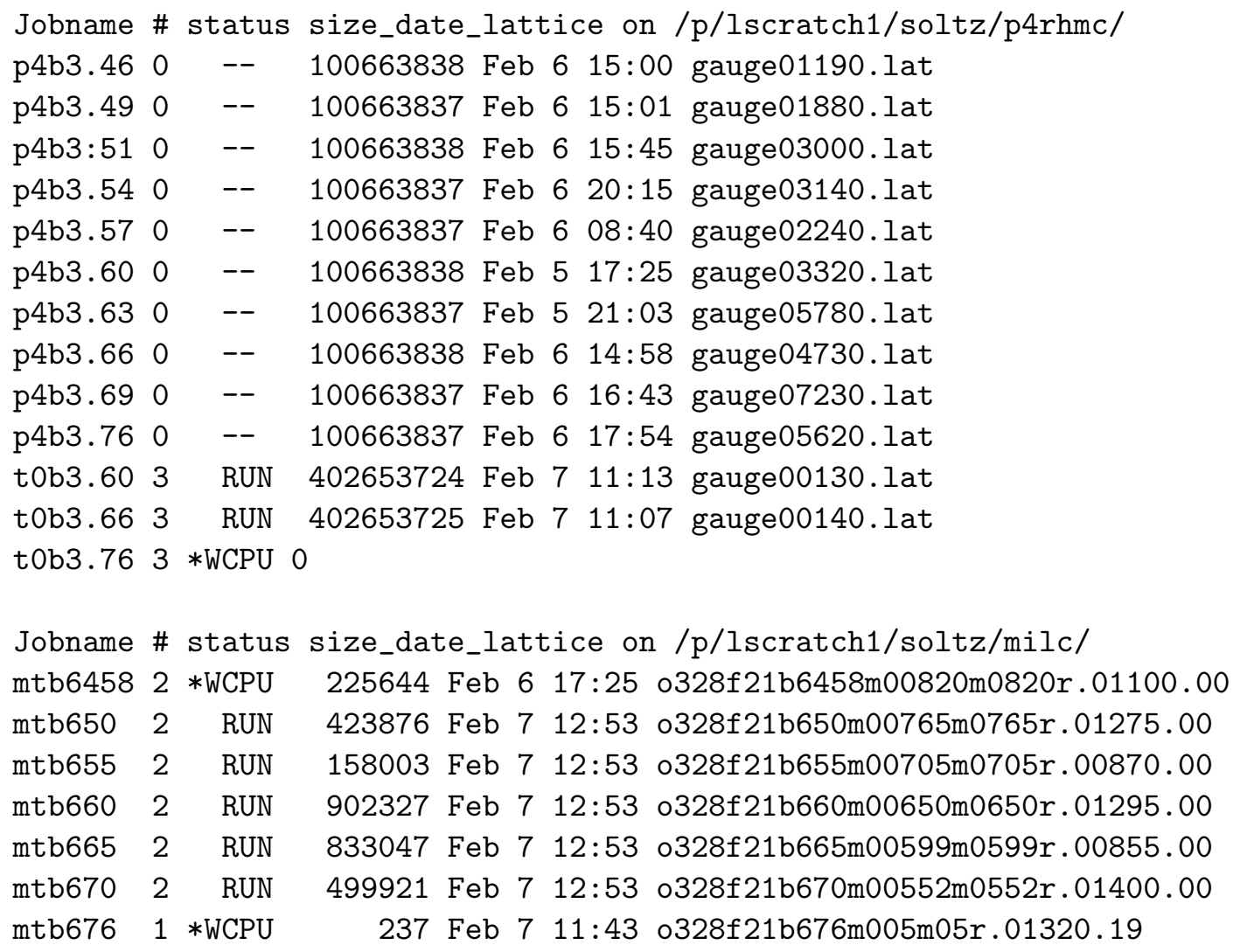




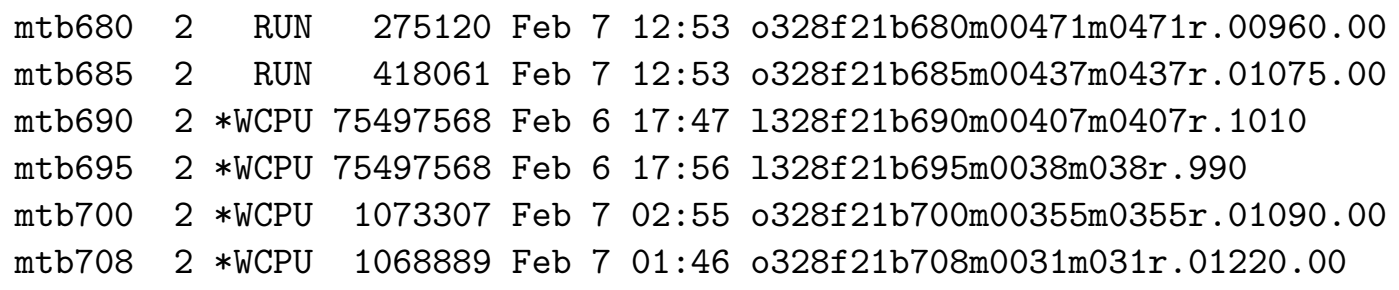

Output of statSet.pl on Thursday, Feb 08, 2007, 8 AM:

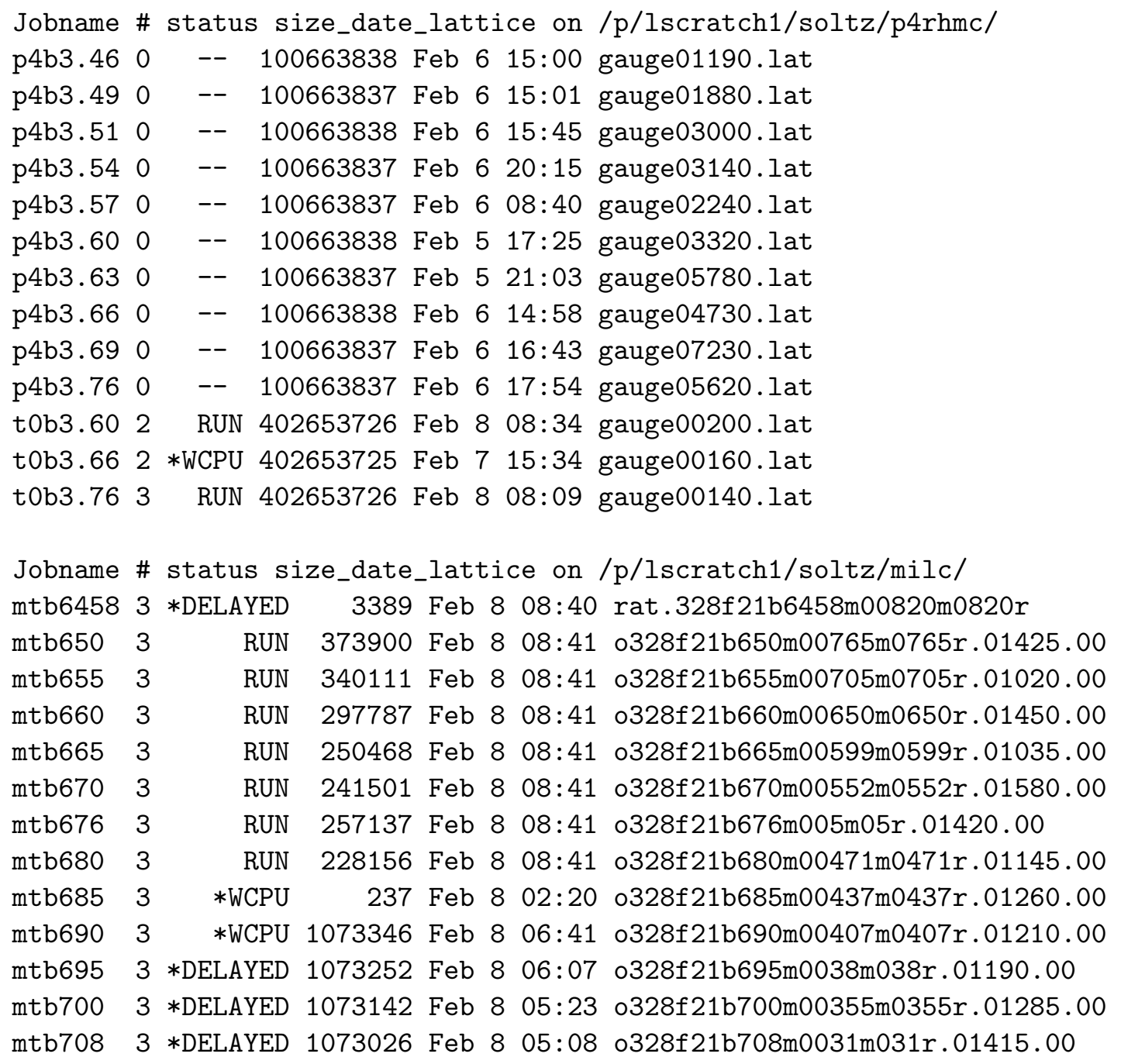




\section{Analysis of $\beta=3.55$ delta-H History}

Previously, the $\beta=3.55$ summary.dat file showed an anomalously large expectation value for $\exp -\Delta H$. Fig. 1 shows this to be due to the values in the very first runs, which were produced when the job was unable to locate the starting thermalized lattice. The data files for these runs were mistakenly copied to the analysis area.

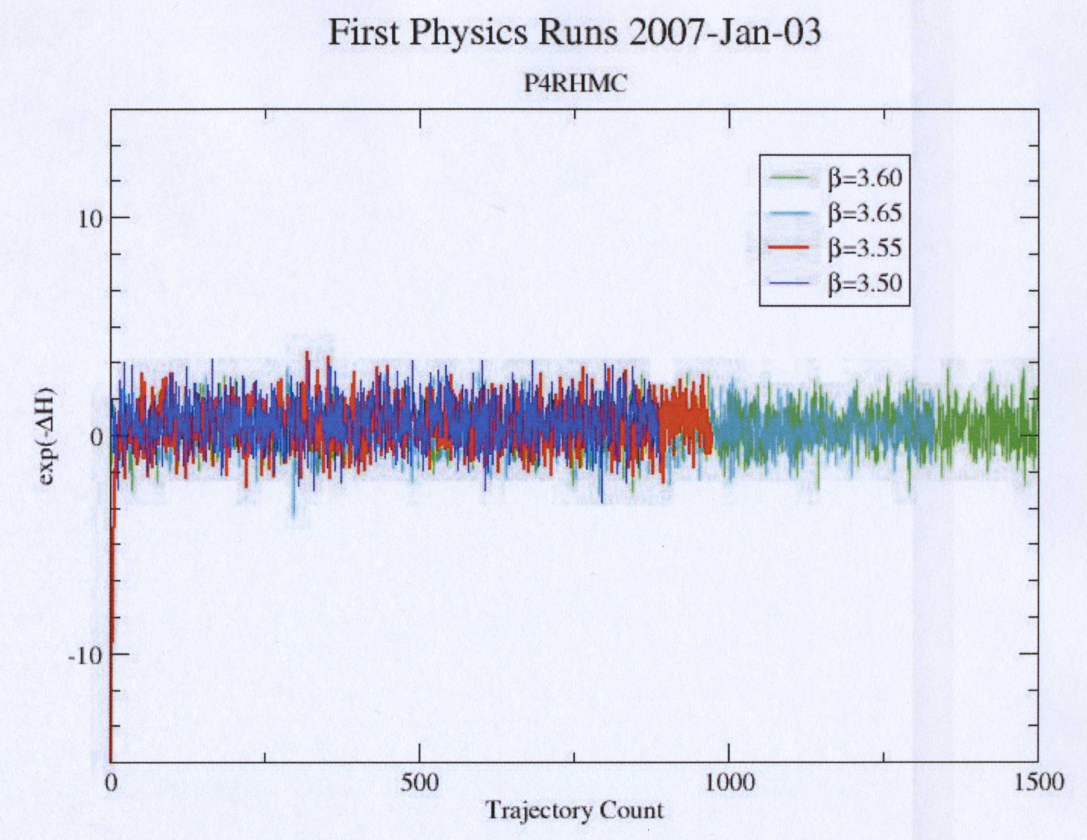

Figure 1: Delta-H history for all $\beta$ values in the first physics runs with p4rhmc. The initial values for $\beta=3.55$ are the result of an error in starting the calcluation.

The following summary file is for $\beta=3.55$ with these initial runs removed.

beta $=3.55$

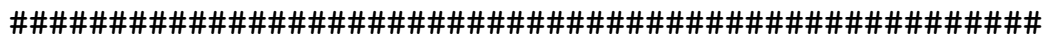




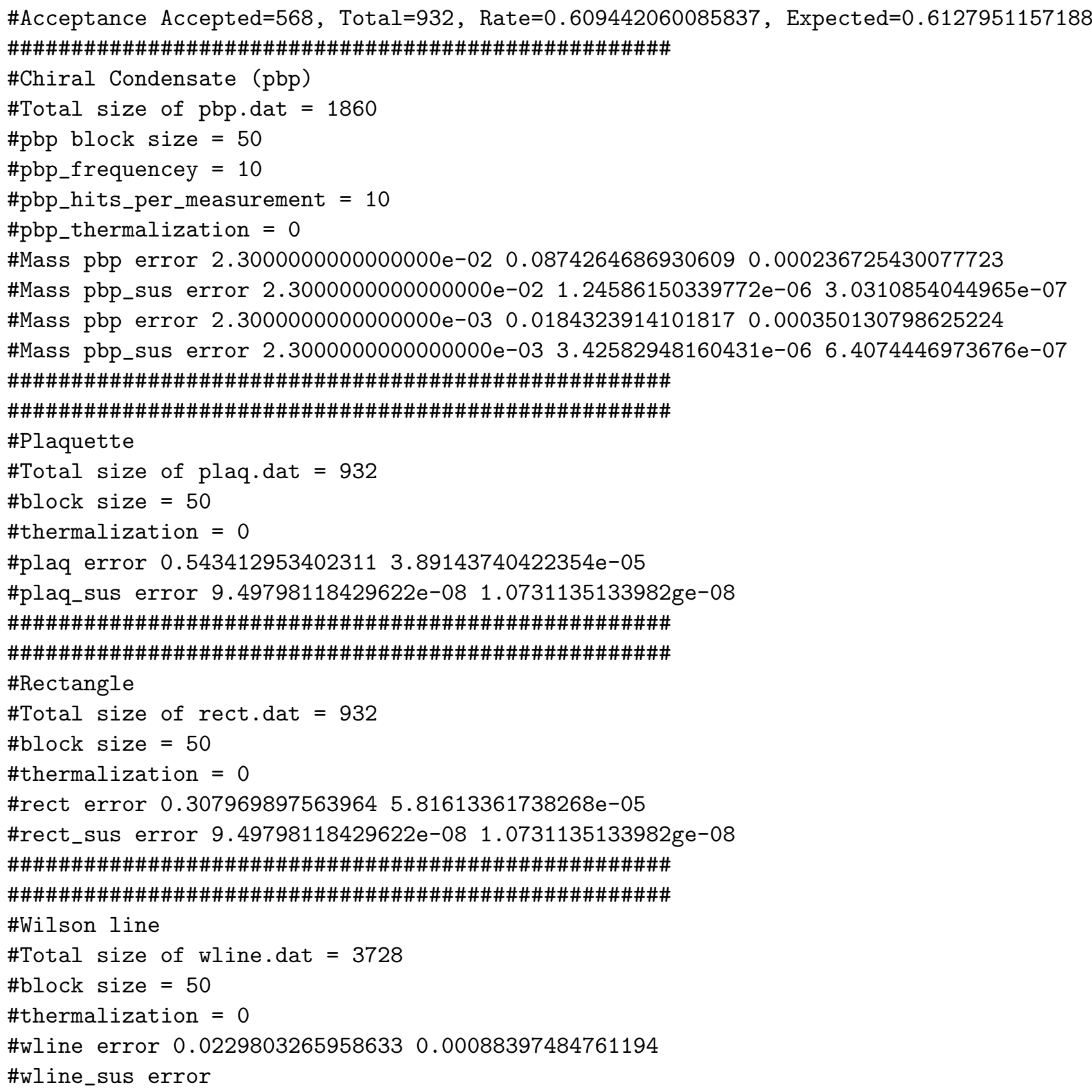




\section{Plaquette History Analysis}

The plaquette history values are used to estimate thermalization times.

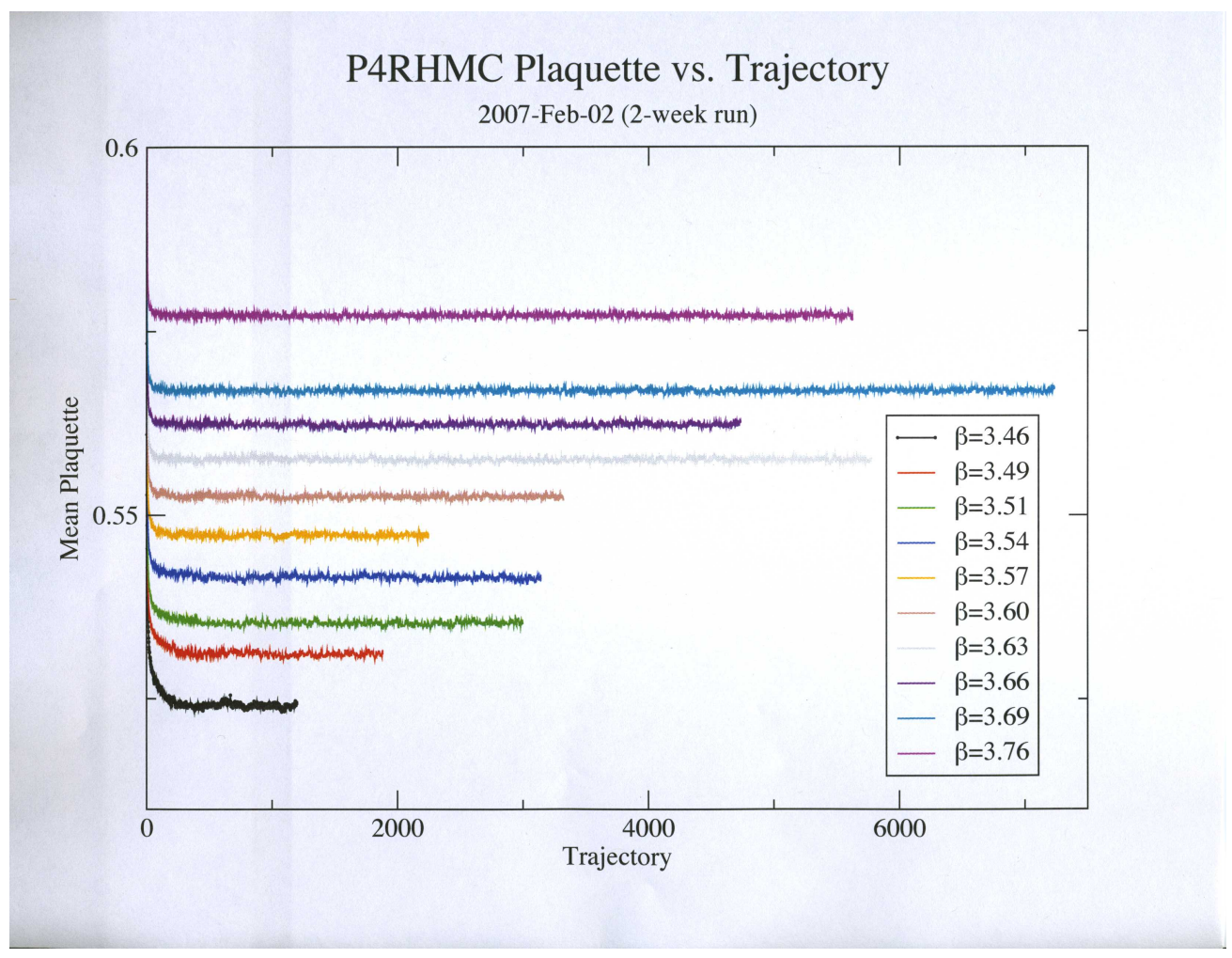

Figure 2: Plaquette histories for $10 \beta$ values for the initial set of $32^{3} x 8$ runs with p4rhmc.

\section{Summary data files}

The following are the summary data files for all $\beta$ values with thermalization cutoff nominally set to 400 .

beta $=3.46$

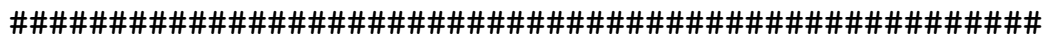

\#Acceptance Accepted $=431$, Total $=798$, Rate $=0.540100250626566$, Expected $=0.532439347744$

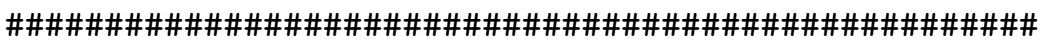




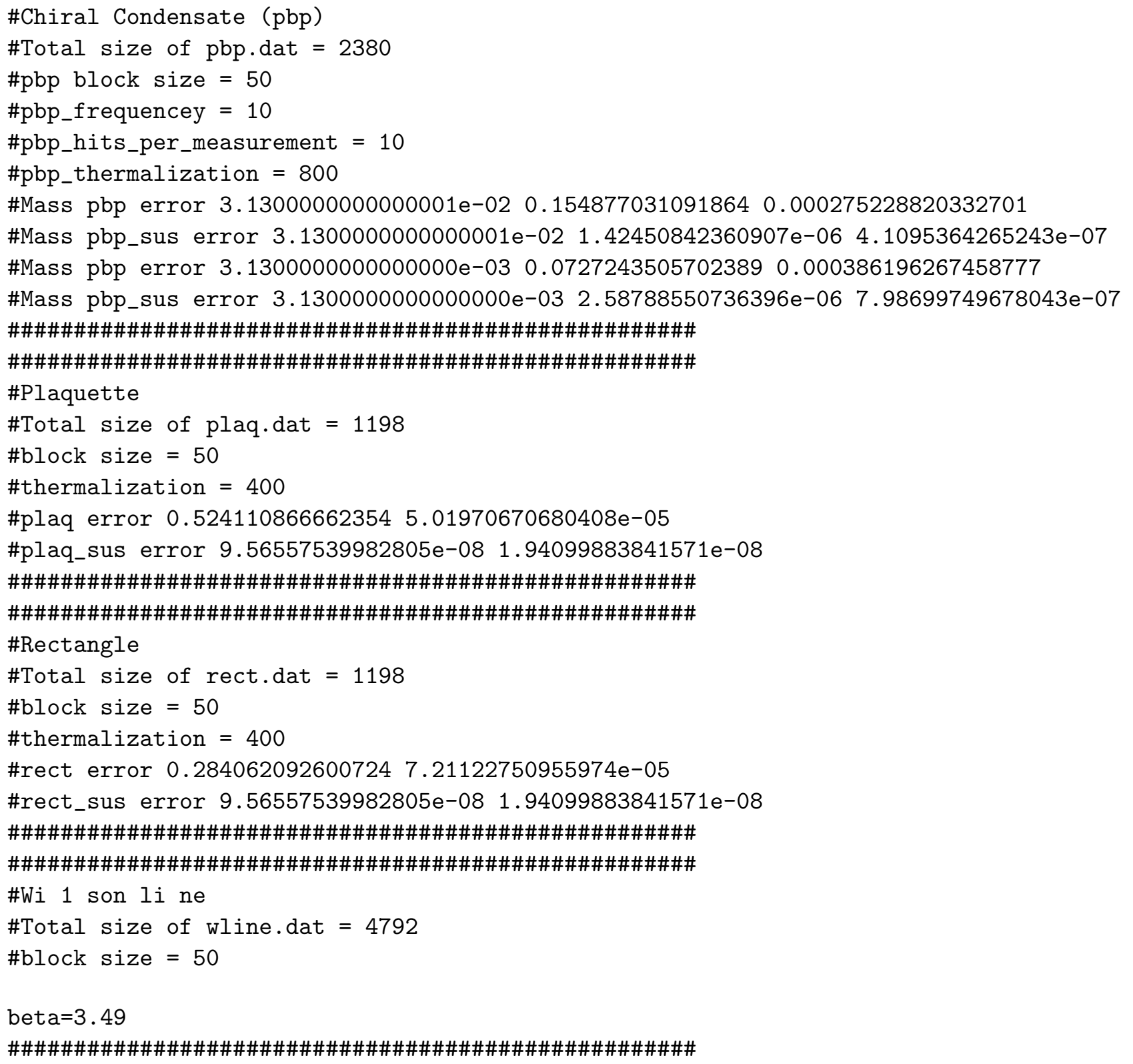




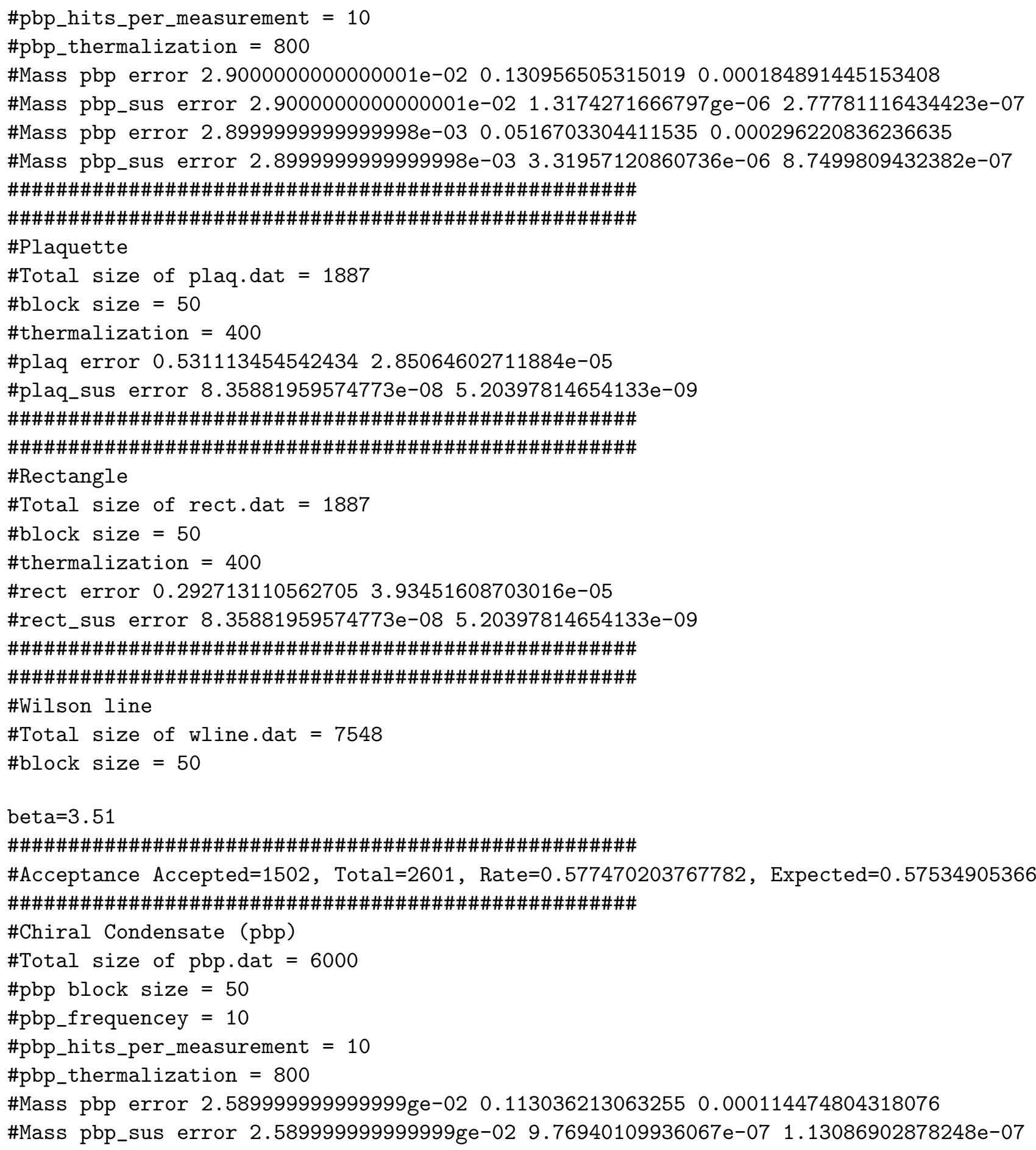


\#Mass pbp error 2.589999999999999ge-03 0.03994209212801520 .000214938433969671 \#Mass pbp_sus error 2.589999999999999ge-03 3.47465478284097e-06 4.05760484671382e-07

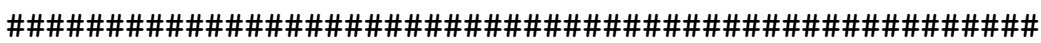

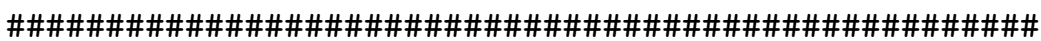
\#Plaquette

\#Total size of plaq.dat $=3001$

$\#$ block size $=50$

\#thermalization $=400$

\#plaq error $0.5353397968282221 .75045465365658 \mathrm{e}-05$

\#plaq_sus error 7.69606817362651e-08 4.8574415767367e-09

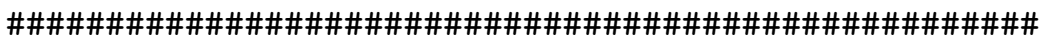

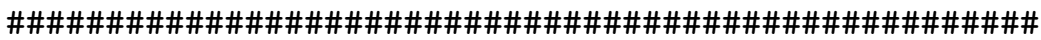

\#Rectangle

\#Total size of rect.dat $=3001$

$\#$ block size $=50$

\#thermalization $=400$

\#rect error $0.29792898883632 .5201369754703 \mathrm{e}-05$

\#rect_sus error 7.69606817362651e-08 4.8574415767367e-09

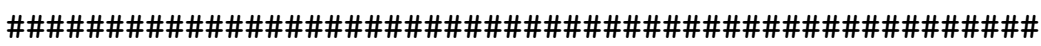

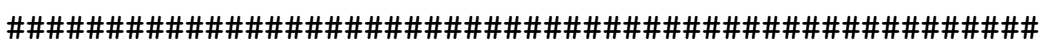

\#Wi 1 son $1 i$ ne

\#Total size of wline.dat $=12004$

$\#$ \#lock size $=50$

$b=3.54$

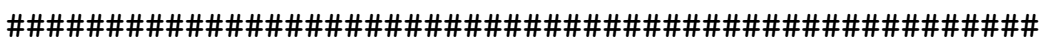

\#Acceptance Accepted=1686, Total=Z746, Rate=0.613983976693372, Expected=0. 6172890304

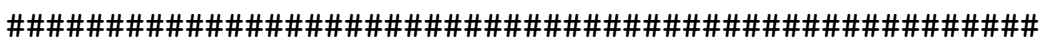

\#Chiral Condensate (pbp)

\#Total size of pbp.dat $=6280$

$\#$ pbp block size $=50$

\#pbp_frequencey $=10$

\#pbp_hits_per_measurement $=10$

\#pbp_thermalization $=800$

\#Mass pbp error 2.4000000000000000e-02 0.09314354334539490 .000170638920465567

\#Mass pbp_sus error 2.4000000000000000e-02 1.78681593188871e-06 2.45285152166955e-07

\#Mass pbp error 2.3999999999999998e-03 0.02119515963778210 .00030749278380982

\#Mass pbp_sus error 2.3999999999999998e-03 6.39794440875484e-06 8.38204282524751e-07

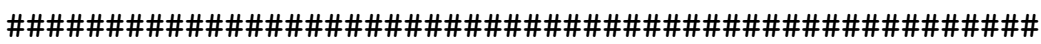

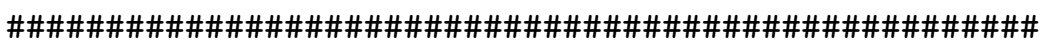




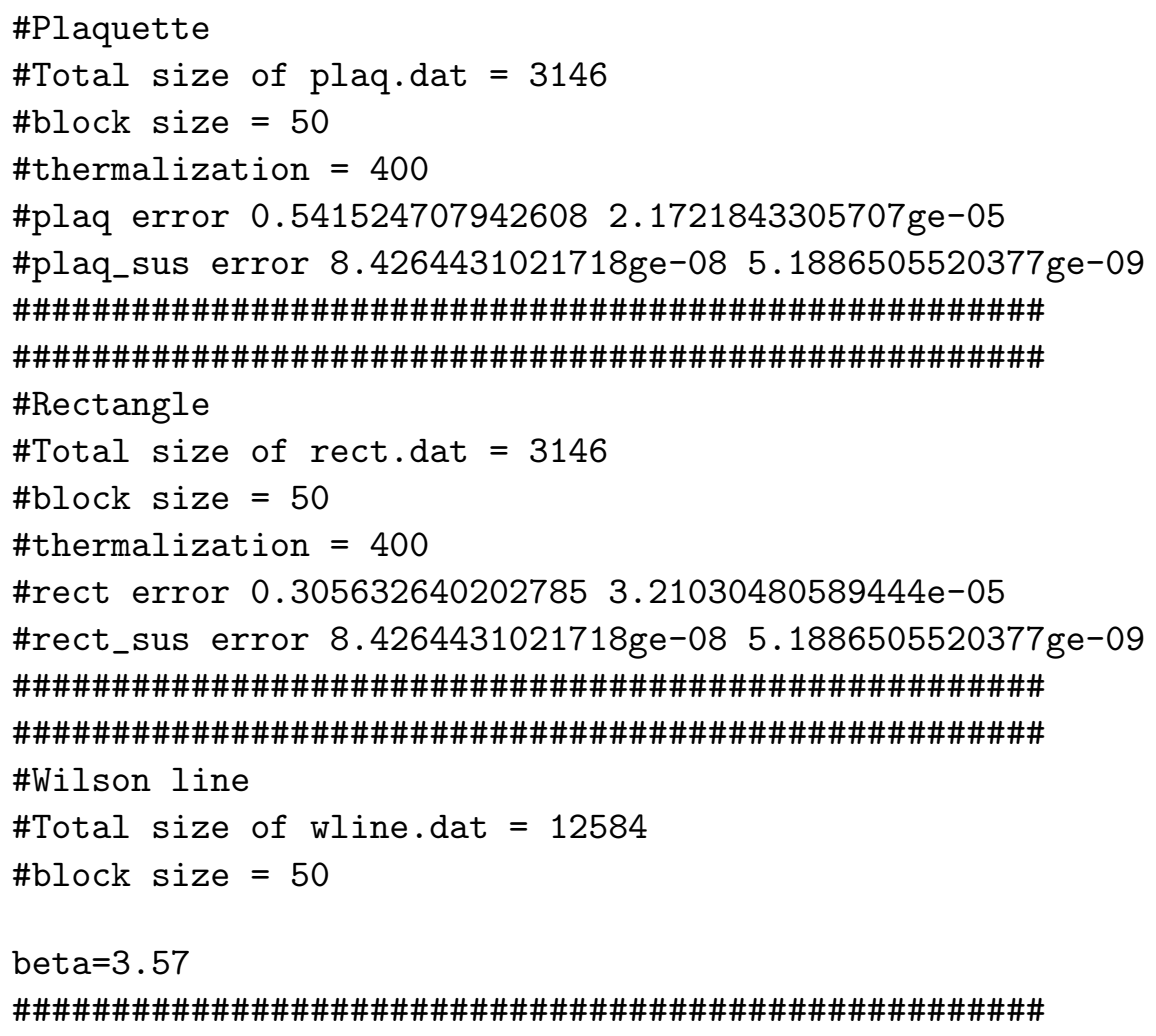


\#plaq error 0.5472846951463462 .0174484632954 ge-05

\#plaq_sus error 7.3057560630269ge-08 4.13491791468014e-09

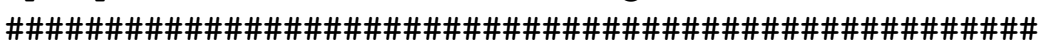

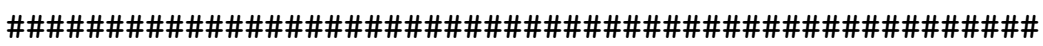

\#Rectangle

\#Total size of rect.dat $=2250$

\#block size $=50$

\#thermalization $=400$

\#rect error 0.3128201491746912 .9179378325158 ge-05

\#rect_sus error 7.3057560630269ge-08 4.13491791468014e-09

\#\#\#\#\#\#\#\#\#\#\#\#\#\#\#\#\#\#\#\#\#\#\#\#\#\#\#\#\#\#\#\#\#\#\#\#\#\#\#\#\#\#\#\#\#\#\#\#

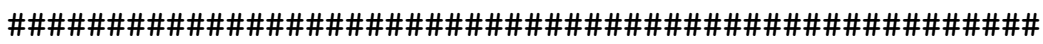

\#Wilson line

\#Total size of wline.dat $=9000$

$\#$ \#lock size $=50$

beta $=3.60$

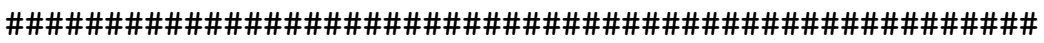

\#Acceptance Accepted $=2083$, Total $=2929$, Rate $=0.711164219870263$, Expected $=0.71707628018$

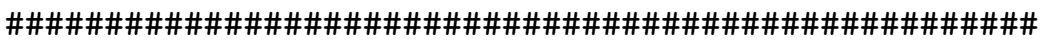

\#Chiral Condensate (pbp)

\#Total size of pbp.dat $=6640$

$\#$ pbp block size $=50$

\#pbp_frequencey $=10$

\#pbp_hits_per_measurement $=10$

\#pbp_thermalization $=800$

\#Mass pbp error 1.9199999999999998e-02 0.0615532435279621 8.31588564428407e-05

\#Mass pbp_sus error 1.9199999999999998e-02 4.93863696607813e-07 6.7410510066550ge-08

\#Mass pbp error . 1.9200000000000000e-03 0.00722918550221107 4.97967917983531e-05

\#Mass pbp_sus error 1.9200000000000000e-03 2.56466108612504e-07 4.02756758232038e-08

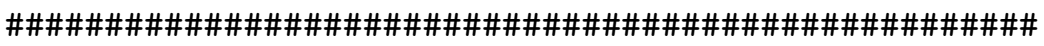

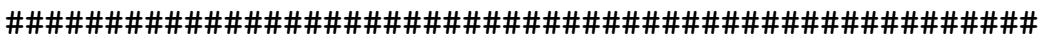

\#Plaquette

\#Total size of plaq.dat $=3329$

$\#$ block size $=50$

\#thermalization $=400$

\#plaq error $0.5525273441097081 .46093164423691 \mathrm{e}-05$

\#plaq_sus error 6.779032376784e-08 3.3077006785081e-09

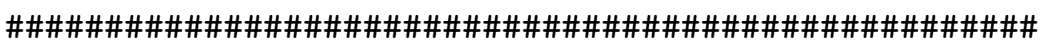

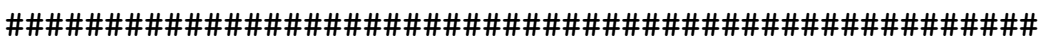




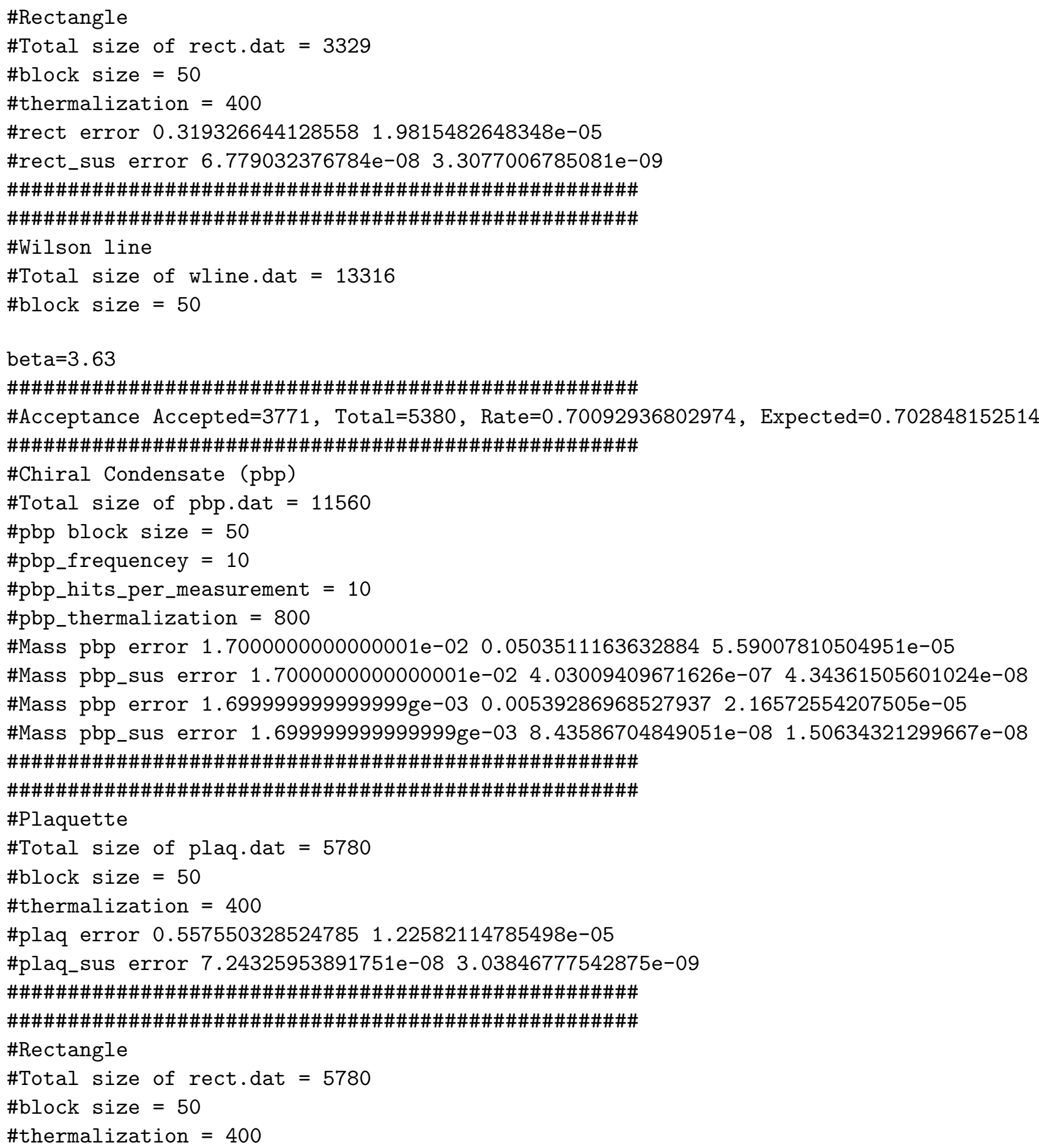




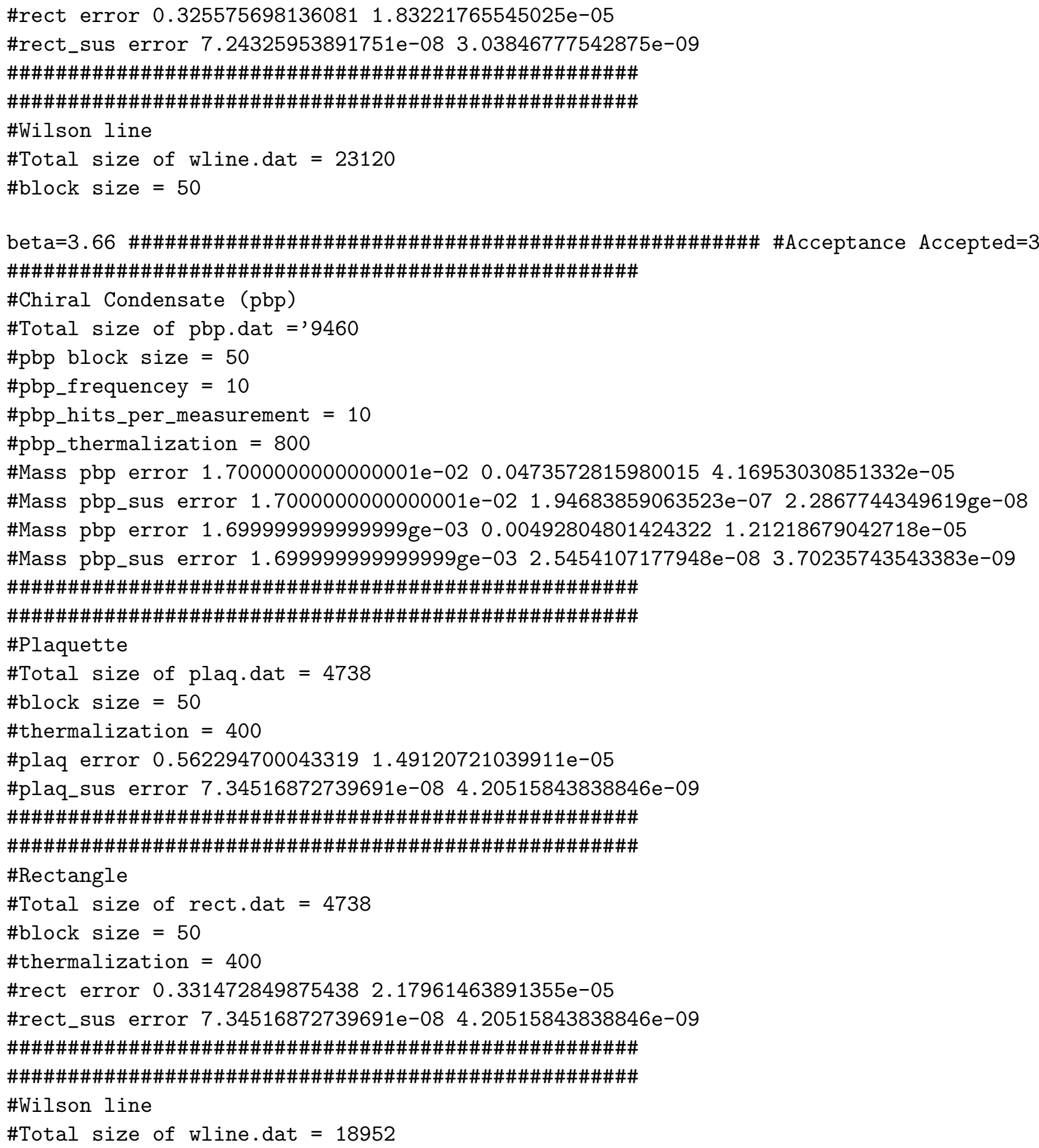


$\#$ block size $=50$

beta=3.69

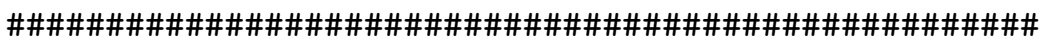

\#Acceptance Accepted $=4694$, Total $=6838$, Rate $=0.686458028663352$, Expected $=0.69092044658$

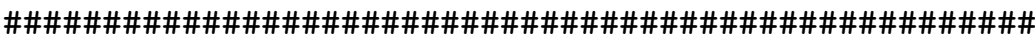

\#Chiral Condensate (pbp)

\#Total size of pbp.dat $=14460$

$\#$ pbp block size $=50$

\#pbp_frequencey $=10$

\#pbp_hits_per_measurement $=10$

\#pbp_thermalization $=800$

\#Mass pbp error 1.499999999999999ge-02 0.0397419877398399 2.22933007422785e-05

\#Mass pbp_sus error 1.499999999999999ge-02 9.87104436320635e-08 9.25518369596677e-09

\#Mass pbp error $1.5000000000000000 \mathrm{e}-030.004053882235739155 .51300481310112 \mathrm{e}-06$

\#Mass pbp_sus error 1.5000000000000000e-03 8.90033977918697e-09 2.15019288186467e-09

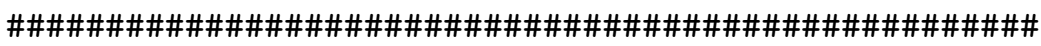

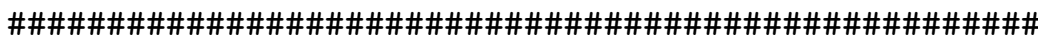

\#Plaquette

\#Total size of plaq.dat $=7238$

$\#$ block size $=50$

\#thermalization $=400$

\#plaq error $0.56691563972838 .45801590902053 \mathrm{e}-06$

\#plaq_sus error 6.20752329879092e-08 2.06678874007656e-09

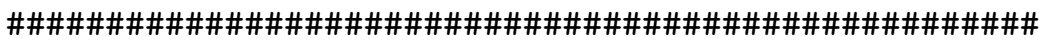

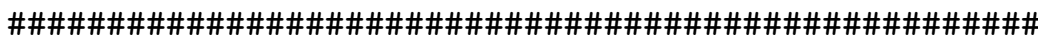

\#Rectangle

\#Total size of rect.dat $=7238$

\#block size $=50$

\#thermalization $=400$

\#rect error 0.337254229945257 $1.27506814428676 \mathrm{e}-05$

\#rect_sus error 6.20752329879092e-08 2.06678874007656e-09

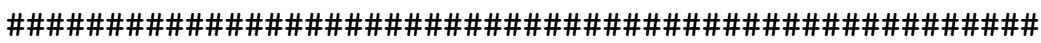

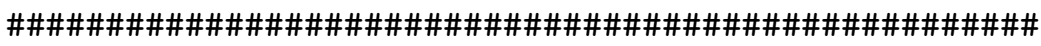

\#Wilson line

\#Total size of wline.dat $=28952$

$\#$ \#lock size $=50$

beta $=3.76$

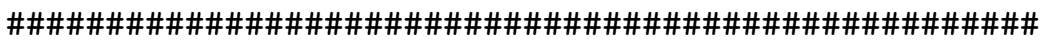




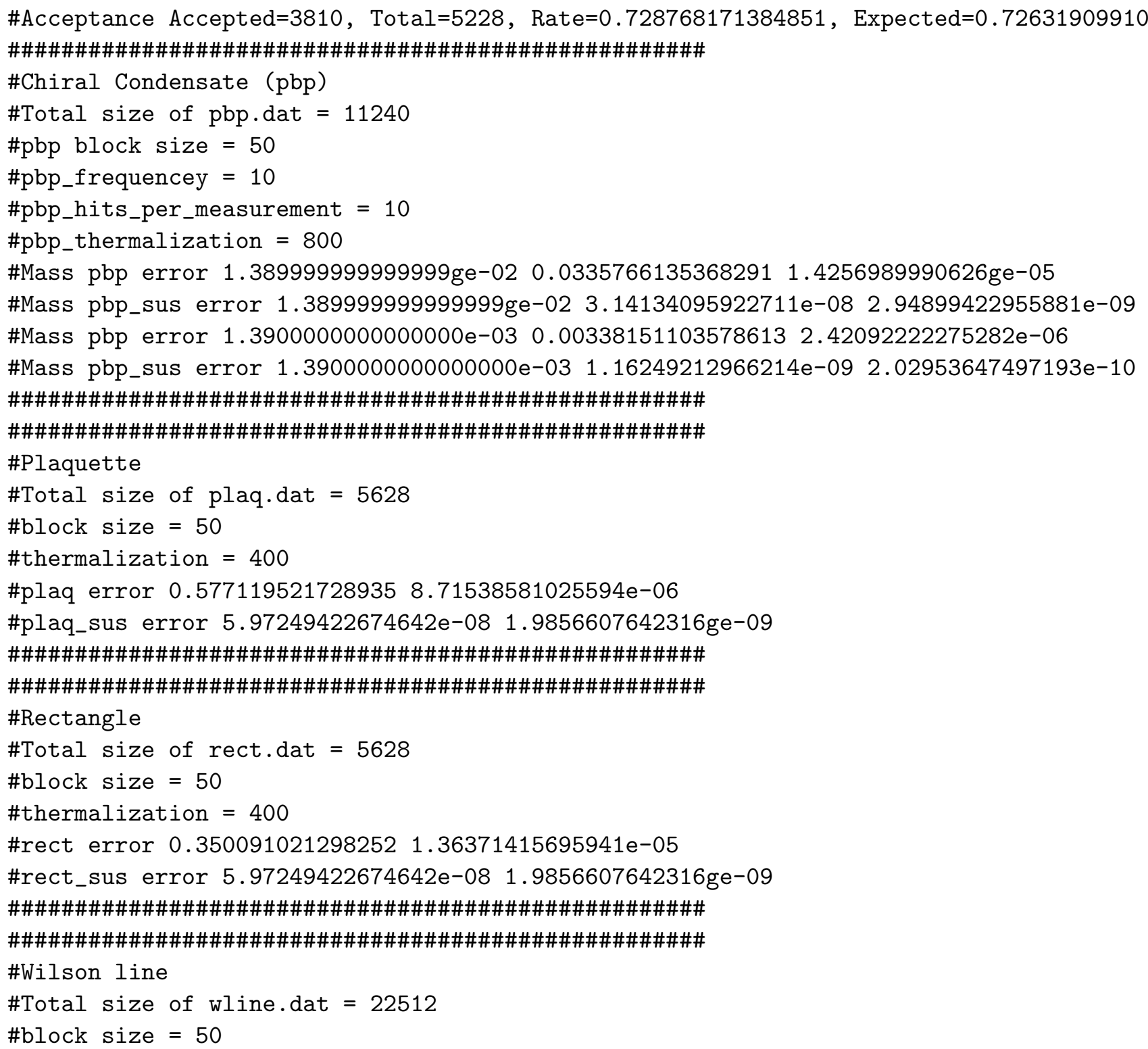

